\title{
Successful Endovascular Stenting of a Bleeding External Iliac Vein Mycotic Aneurysm: A Case Report
}

\section{Rupal Parikh ( $\square$ rupalparikh90@gmail.com )}

University of Pennsylvania Perelman School of Medicine https://orcid.org/0000-0002-9389-4167

Shiyi Li

Hospital of the University of Pennsylvania

Christopher Shackles

Holy Name Medical Center

Tamim Khaddash

Hospital of the University of Pennsylvania

\section{Research Article}

Keywords: hemorrhage, vein, endovascular treatment, stenting, venous intervention

Posted Date: April 22nd, 2021

DOl: https://doi.org/10.21203/rs.3.rs-416167/v1

License: @ (i) This work is licensed under a Creative Commons Attribution 4.0 International License. Read Full License 


\section{Abstract}

Background: Mycotic aneurysms are rare vascular lesions, occurring in $0.6-2 \%$ of arterial aneurysms but with no reported venous cases. Venous aneurysms unrelated to an underlying infectious process have been previously described and are typically surgically repaired due to risk of thromboembolic events. Only a few reported cases have been successfully treated with endovascular repair.

Case presentation: This case reports a bleeding external iliac vein mycotic aneurysm secondary to erosion of a chronic pelvic abscess, successfully treated with endovascular stenting.

Conclusion: Venous aneurysms are uncommon vascular lesions which have historically been treated with open surgical repair. Given the lower degree of procedural morbidity, endovascular management of these lesions may be an effective option in the appropriate setting.

\section{Background}

Arising either from vessel wall damage from a primary infection or secondary infection of a preexisting aneurysm, mycotic aneurysms occur in approximately $0.6-2 \%$ of arterial aneurysms (1). Given the significant morbidity and mortality associated with mycotic arterial aneurysms, standard of care consists of aggressive antibiotic therapy and surgical debridement/reconstruction (2-3). With recent advancements however, minimally-invasive management of mycotic arterial aneurysms is becoming an increasingly more common treatment option (4-5). Furthermore, compared to arterial aneurysms, venous aneurysms are rare and more indolent in presentation. Due to the risk of thromboembolic events, recent literature supports treatment of lower extremity deep venous aneurysms, particularly popliteal vein aneurysms $(1,6)$. While isolated cases of iliac artery mycotic aneurysms are known, no cases involving the iliac vein have been reported and thus management guidelines for these patients are limited (7-9). This case reports a bleeding external iliac vein (EIV) mycotic aneurysm secondary to erosion of a pelvic abscess into the EIV, successfully repaired with endovascular stenting.

\section{Case Presentation}

A 34-year-old female with a history of stage IIB cervical squamous cell carcinoma status post pelvic exenteration and external beam radiation, complicated by a pelvic abscess and chronic drainage catheter, was referred to Interventional Radiology (IR) for management of bleeding around and into the pelvic drain. Laboratory evaluation was significant for an acute $5.1 \mathrm{~g} / \mathrm{dL}$ and $15 \%$ decrease in hemoglobin and hematocrit, respectively. Multiphasic computed tomography (CT) of the abdomen/pelvis demonstrated interval development of hyperattenuation in the chronic pelvic collection, adjacent to a new sac-like dilation of the right EIV, concerning for vascular erosion by the chronic pelvic abscess (Fig. 1). The patient was transfused with two units of packed red blood cells with an appropriate increase in hemoglobin/hematocrit. The patient remained hemodynamically stable and was continued on her outpatient intravenous (IV) antibiotic regimen (daptomycin, cetazidime-avibactam, and metronidazole) 
for recent vancomycin-resistant enterococcal bacteremia and chronic pelvic abscess. The patient was subsequently brought to IR.

\section{Procedure}

The right common femoral vein (CFV) was accessed using standard micropuncture technique. Digital subtraction venography (DSV) of the right CFV to the level of the infrarenal IVC was performed through a transitional sheath, which demonstrated a focal sac-like outpouching arising from the right EIV with eventual contrast extravasation, corresponding to the area of suspected erosion and consequent irregularity of the right EIV seen on cross sectional imaging (Fig. 2). The transitional sheath was then exchanged for a $13 \mathrm{~cm}$ long, 12 French ( $\mathrm{Fr}$ ) vascular sheath and a marking catheter was advanced into the right EIV. A $13 \mathrm{~mm} \times 5 \mathrm{~cm}$ Viabahn stent graft was advanced to the targeted area of stenting, and DSV demonstrating contrast extravasation associated with the pseudoaneurysm was performed prior to deployment. The stent was immediately deployed into the EIV at the level of the mycotic venous aneurysm and postdilated to $14 \mathrm{~mm}$. Post deployment DSV demonstrated an area of contrast extravasation (Fig. 3A), along the inferior margin of the newly deployed stent. An additional $11 \mathrm{~mm} \times 10$ $\mathrm{cm}$ Viabahn stent graft was placed and postdilated to $10 \mathrm{~mm}$. Completion DSV demonstrated no evidence of persistent hemorrhage, with brisk inline flow throughout the stent complex into the IVC (Fig. 3B). The sheath was removed and manual pressure was maintained until hemostasis was achieved.

To exclude any superimposed arterial bleeding, the decision was made to study the ipsilateral arterial system. The right common femoral artery (CFA) was then accessed using standard micropuncture technique. A $5 \mathrm{Fr}$ straight flush catheter was advanced "bareback" without a sheath with its tip in the proximal right common iliac artery and digital subtraction arteriography (DSA) of the right external iliac artery was performed in multiple projections with no contrast extravasation to suggest superimposed arterial injury (Fig. 4).

\section{Post-procedure course}

The patient tolerated the procedure well and remained hemodynamically stable throughout her hospital course. On post-procedure day 2 , she was discharged to home on a one-week course of IV antibiotics with infectious disease follow-up and plan for lifelong antimicrobial prophylaxis due to her chronic pelvic abscess and concern for possible stent graft superinfection.

One month post-procedure, the patient presented to the emergency department with right thigh swelling and pelvic pain. Contrast-enhanced CT abdomen/pelvis at the time demonstrated new occlusive right femoral venous thrombosis extending through the stent to the level of the common iliac vein. Given concern for superinfection of the occlusive thrombus and increased procedural risk of septic pulmonary emboli, the patient was started on therapeutic anticoagulation. She subsequently developed persistent hematuria, at which time a convertible IVC filter was placed via the left femoral approach and therapeutic anticoagulation was discontinued with resolution of hematuria. 


\section{Conclusions}

This case reports a bleeding mycotic venous aneurysm secondary to erosion of a chronic pelvic abscess into the EIV, successfully treated with endovascular stenting. Venous aneurysms are rare vascular lesions with limited published management guidelines. While imaging surveillance is recommended for thoracic and upper extremity aneurysms, open surgical repair, such as aneurysmectomy, venorrhaphy, and end-toend anastomosis, is indicated for lower extremity aneurysms due to a higher risk of thromboembolic events (6). Only a few cases of endovascular treatment of mycotic aneurysms have been reported (910). Furthermore, venography and endovascular management of mycotic venous aneurysms have not been reported. The iliac veins, in particular, may be more amenable to stenting given their deep and relatively fixed locations. The present report demonstrates that endovascular stenting is a safe and effective option in patients with EIV mycotic aneurysms, especially in patients with complex surgical histories, predisposing to adhesive disease. While the result was not durable in terms of long-term patency, life threatening hemorrhage was prevented and the patient's life was extended.

Venous aneurysms are uncommon vascular lesions which have historically been treated with open surgical repair. The present case reports a mycotic EIV aneurysm, treated safely and effectively with endovascular stenting. Given the lower degree of procedural morbidity, endovascular management of these lesions may be considered a viable option to prevent life threatening hemorrhage.

\section{Declarations}

1. Ethics approval and consent to participate: This study has been reviewed by the institutional review board and the need for ethics approval was waived.

2. Consent for publication: Written informed consent was obtained from the patient for publication of this case report and any accompanying images.

3. Availability of data and materials: Not applicable.

4. Competing interests: Not applicable.

5. Funding: This study was not supported by any funding.

6. Authors contributions: RSP and SL performed the chart review and were major contributors in writing the manuscript. CS and TK were major contributors in reviewing the manuscript. TK was a major contributor in mentoring the creation of this manuscript. All authors read and approved the final manuscript.

7. Acknowledgements: Not applicable.

8. Authors' information: See Title page.

\section{References}

1. Patel R, Hanish S, Baril D, Woo K, Lawrence P. Contemporary management of lower extremity venous aneurysms. J Vasc Surg Venous Lymphat Disord. 2019;7(6):860-864. doi: 
10.1016/j.jvsv.2019.06.017

2. Kim, Y. Infected aneurysm: current management. Ann Vasc Dis. 2010;3(1):7-15. doi: 10.3400/avd.AVDctiia09003

3. Sörelius K, Budtz-Lilly J, Mani K, Wanhainen A. Systematic review of the management of mycotic aortic aneurysms. Eur J Vasc Endovasc Surg. 2019;58(3):426-435.

https://doi.org/10.1016/j.ejvs.2019.05.004

4. Sörelius K, Mani K, Björck M, et al. Endovascular treatment of mycotic aortic aneurysms: a European multicenter study. Circulation. 2014;130(24): 2136-2142. doi:

10.1161/CIRCULATIONAHA.114.009481

5. Kan CD, Lee HL, Yang, YJ. Outcome after endovascular stent graft treatment for mycotic aortic aneurysm: a systematic review. J Vasc Surg. 2007;46(5):906-912. doi: 10.1016/j.jvs.2007.07.025

6. Teter KA, Maldonado TM, Adelman MA. A systematic review of venous aneurysms by anatomic location. J Vasc Surg Venous Lymphat Disord. 2018;6(3):408-413. doi:10.1016/j.jvsv.2017.11.014

7. Salam A, Khan I, Sonawalla A, Fatimi S. Rare mycotic aneurysms of internal jugular vein and innominate vein secondary to untreated parapharyngeal abscess: A case report. Ann Med Surg (Lond). 2017;19:62-64. doi:10.1016/j.amsu.2017.05.011

8. Brant-Zawadzki P, Kinikini D, Kraiss LW. Deep leg vein reconstruction for an isolated mycotic common iliac artery aneurysm in an HIV-positive patient. Vascular. 2007;15(2):98-101. doi:10.2310/6670.2007.00014

9. Chandler BT, Ryer EJ, Keyser BM, Elmore JR. A hybrid approach to appendicitis with right external iliac artery pseudo aneurysm: A case report. Int J Surg Case Rep. 2017;33:99-101. doi:10.1016/j.ijscr.2017.03.001

10. San Norberto EM, Gutiérrez VM, Revilla A, Vaquero C. Subclavian venous aneurysm: endovascular treatment. J Vasc Interv Radiol. 2010;21(8):1306-1308. doi:10.1016/j.jvir.2010.02.029

\section{Figures}




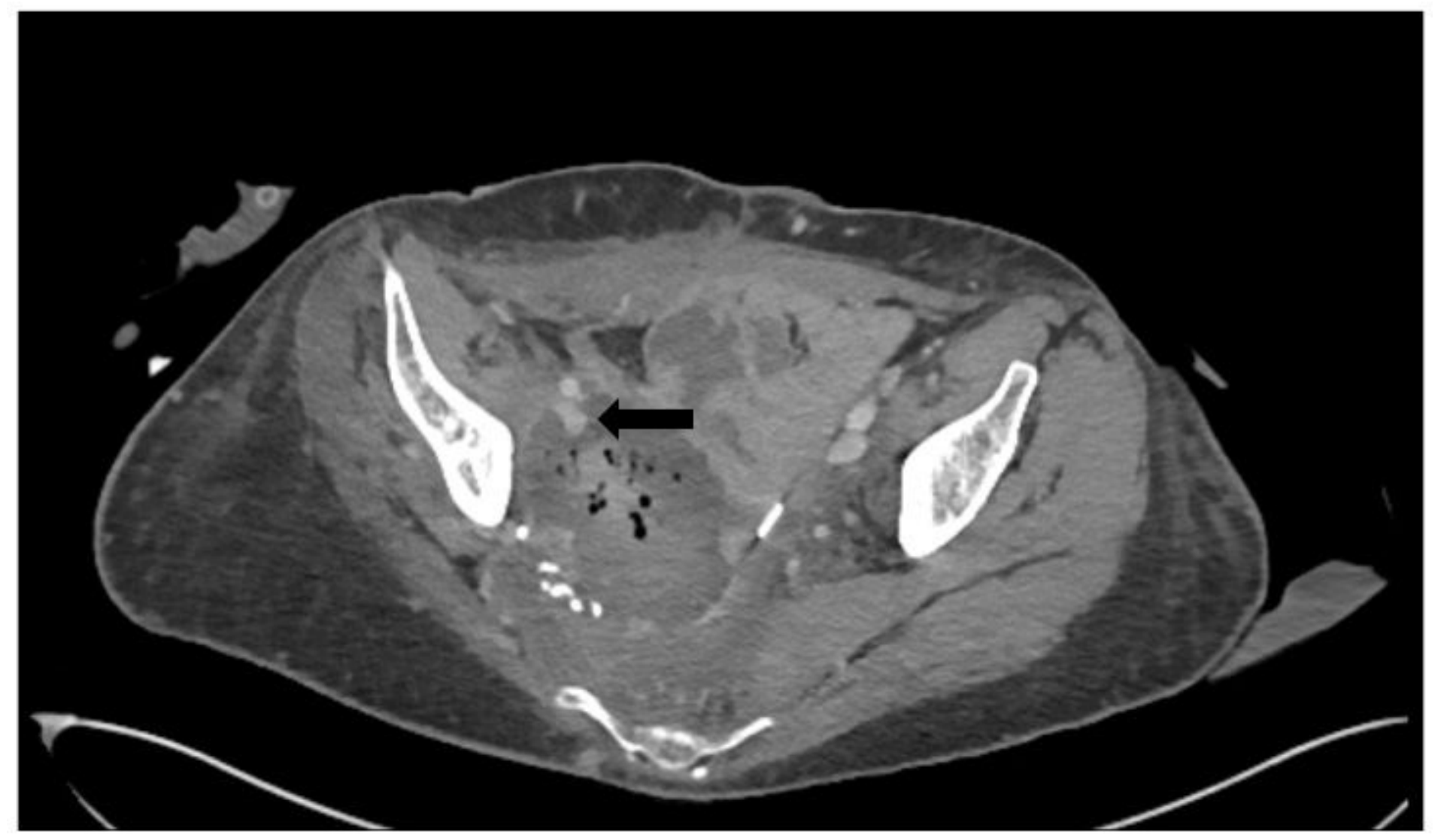

\section{Figure 1}

Venous phase imaging of multiphasic CT-abdomen/pelvis demonstrates interval development of hyperattenuation in the chronic pelvic collection with locules of air, adjacent to a new sac-like dilation arising from the right EIV (black arrow), concerning for vascular erosion by the chronic pelvic abscess. 


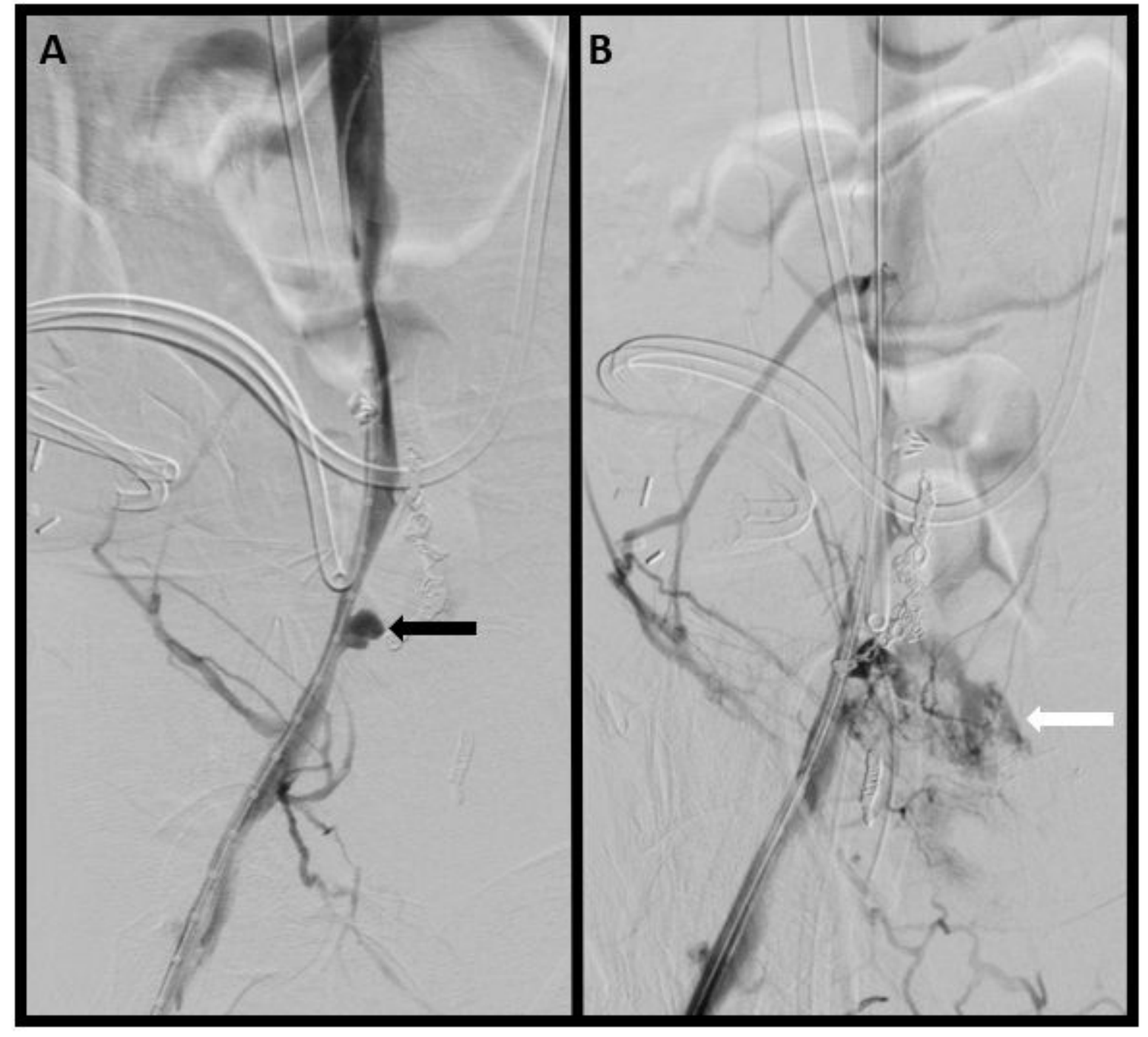

Figure 2

Initial venogram $(A)$ of the right EIV demonstrates a focal sac-like outpouching arising from the right EIV (black arrow). Subsequent venogram (B) depicts contrast extravasation (white arrow) corresponding to the area of suspected erosion and consequent irregularity of the right EIV seen on cross sectional imaging. 


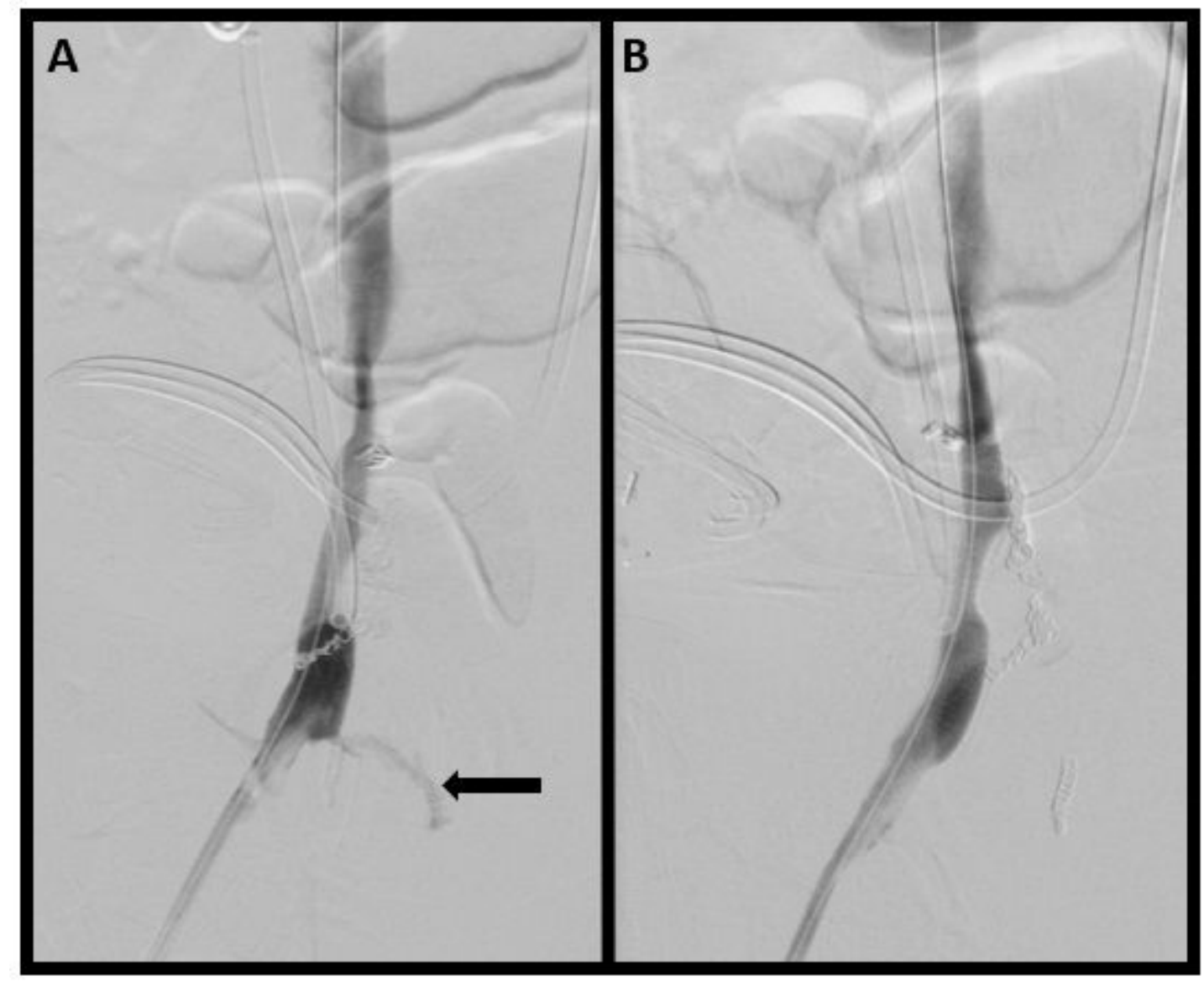

Figure 3

Placement of a $13 \mathrm{~mm} \times 5 \mathrm{~cm}$ Viabahn stent graft into the EIV, postdilated to $14 \mathrm{~mm}$, though with persistent contrast extravasation (A, black arrow). Placement of an additional $11 \mathrm{~mm} \times 10 \mathrm{~cm}$ Viabahn stent graft into the EIV, postdilated to $10 \mathrm{~mm}$, with no persistent extravasation (B). 


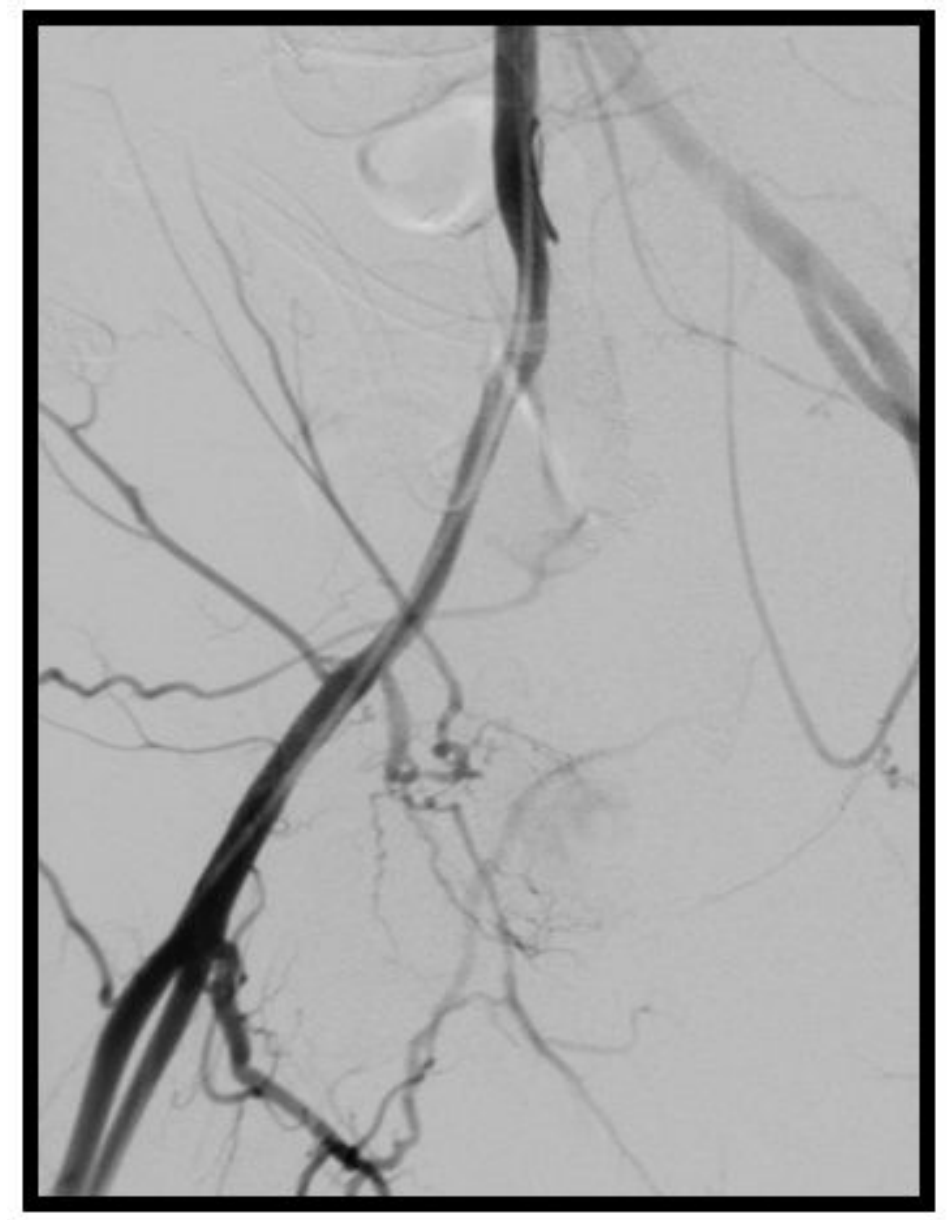

\section{Figure 4}

Right external iliac arteriography demonstrates no contrast extravasation to suggest superimposed arterial injury. 